\title{
A changing inner radius in the accretion disc of Q0056-363?
}

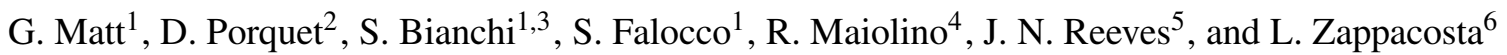 \\ 1 Dipartimento di Fisica, Università degli Studi Roma Tre, via della Vasca Navale 84, 00146 Roma, Italy \\ e-mail: matt@fis.uniroma3.it \\ 2 Max-Planck-Institut für extraterrestrische Physik, Postfach 1312, 85741 Garching, Germany \\ 3 XMM-Newton Science Operation Center, ESAC/ESA, Apartado 50727, 28080 Madrid, Spain \\ 4 INAF - Osservatorio Astrofisico di Arcetri, Largo Fermi 5, 50125 Firenze, Italy \\ 5 Laboratory for High Energy Astrophysics, NASA/GSFC, Greenbelt Road, Greenbelt, MD 20771, USA \\ ${ }^{6}$ Department of Physics and Astronomy, University of California, Irvine, 4129 Frederick Reines Hall, Irvine, \\ CA 92697-4575, USA
}

Received 13 December 2004 / Accepted 15 February 2005

\begin{abstract}
Q0056-363 is the most powerful X-ray quasar known to exhibit a broad, likely relativistic iron line (Porquet \& Reeves 2003, A\&A, 408, 119). It has been observed twice by XMM-Newton, three and half years apart (July 2000 and December 2003). In the second observation, the UV and soft X-ray fluxes were fainter, the hard X-ray power law flatter, and the iron line equivalent width (EW) smaller than in the 2000 observation. These variations can all be explained, at least qualitatively, if the disc is truncated in the second observation. We report also on the possible detection of a transient, redshifted iron absorption line during the 2003 observation.
\end{abstract}

Key words. accretion, accretion disks - galaxies: active - X-rays: galaxies - galaxies: quasars: individual: Q0056-363

\section{Introduction}

The geometrical and physical properties of the inner regions of the accretion discs around the supermassive Black Holes in Active Galactic Nuclei are still largely unknown, despite many theoretical and observational efforts. While there is wide consensus that Comptonization of thermal disc emission by hot electrons is the main mechanism of production of X-rays, the details of this process are by no means clear. The geometry of the inner accretion disc regions, and their very existence, are also matter of debate. While in a few sources relativistic iron line profiles are clearly observed (e.g. Fabian et al. 2002; Turner et al. 2002), implying that the accretion disc is probably extending down to the innermost stable orbit (i.e. $1.23 r_{\mathrm{g}}$ if $a=1$, or $6 r_{\mathrm{g}}$ if $a=0$, where $r_{\mathrm{g}}=G M / c^{2}$ and $a$ is the Black Hole angular momentum per unit mass), in many sources only "narrow" (i.e. unresolved) iron components are present (e.g. Bianchi et al. 2004, and references therein). These narrow lines probably originate in distant matter, like the BLR or the torus, even if in many cases an origin in the outer regions of the accretion disc cannot at present be ruled out.

The reason why in many sources there is no evidence for the presence of the innermost regions of the accretion disc is unclear. One possible explanation is ionization of the matter. If the matter is mildly (or very highly) ionized, iron line emission is efficiently suppressed (Matt et al. 1996). Of course, ionization is expected to be higher in the innermost regions, so mainly suppressing the broader components of the iron line.
Another possibility is disc truncation. In Galactic Black Hole systems, disc truncation is believed to play an essential role in explaining the spectral differences between Soft and Hard states, the main physical parameter likely being the accretion rate (e.g. Done \& Gierlinski 2004; Fender et al. 2004, and references therein). In the Hard state, the disc is truncated while, in the Soft state, it extends down to the innermost stable orbit. An Intermediate state occurs during the transitions between the two states. The disc emptying and refilling is associated with matter ejection, as signalled by radio emission. The radio properties in fact also changes dramatically from state to state. In the Hard state, there is evidence for a steady jet, emitting strongly at radio wavelenghts via synchrotron radiation. Matter ejection is quenched in the High state, while in the Intermediate state the ejection may be present, but often in form of discrete blobs. The disc emptying and refilling may occur on very different time scales, from seconds (as in GRS 1915+105, Belloni et al. 1997, where probably only a limited part of the cycle, i.e. between Intermediate and High states, is covered: Fender et al. 2004) to months. A similar situation may well occur in AGN, and indeed Marscher et al. (2002) have found that the superluminal Seyfert 1 galaxy 3C120, when observed simultaneously in radio and X-rays, behaves similarly to GRS 1915+105, even if, as obvious, on longer time scales. Disc truncation has also been invoked by Zdziarski et al. (1999) and Lubinski \& Zdziarski (2001) to explain the observed correlation between the X-ray spectral slope and both the amount of Compton reflection and the $E W$ (and width) of the iron line. In fact, if the 
Table 1. The best fit parameters for the 2000 and 2003 observations. The model consists of two power laws and a Gaussian line centred at $6.4 \mathrm{keV}$ (rest frame).

\begin{tabular}{|c|c|c|c|c|c|c|c|}
\hline & $\begin{array}{c}\text { FLUX (UWV2) } \\
\left(\mathrm{erg} \mathrm{cm}^{-2} \mathrm{~s}^{-1} \mathrm{~A}^{-1}\right)\end{array}$ & $\begin{array}{c}\text { FLUX }(0.5-2 \mathrm{keV}) \\
\left(\mathrm{erg} \mathrm{cm}^{-2} \mathrm{~s}^{-1}\right)\end{array}$ & $\begin{array}{c}\text { FLUX (2-10 keV) } \\
\left(\mathrm{erg} \mathrm{cm}^{-2} \mathrm{~s}^{-1}\right)\end{array}$ & $\overline{\Gamma_{\text {soft }}}$ & $\overline{\Gamma_{\text {hard }}}$ & $\begin{array}{l}\sigma_{\mathrm{K} \alpha} \\
(\mathrm{eV})\end{array}$ & $\begin{array}{c}E W_{\mathrm{K} \alpha} \\
(\mathrm{eV})\end{array}$ \\
\hline 2000 & $2.11 \times 10^{-14}$ & $3.40 \times 10^{-12}$ & $2.96 \times 10^{-12}$ & $3.35_{-0.14}^{+0.46}$ & $1.87_{-0.06}^{+0.17}$ & $270_{-130}^{+180}$ & $266_{-99}^{+134}$ \\
\hline 2003 & $1.35 \times 10^{-14}$ & $3.02 \times 10^{-12}$ & $2.90 \times 10^{-12}$ & $2.87_{-0.02}^{+0.02}$ & $1.61_{-0.02}^{+0.03}$ & $250_{-100}^{+100}$ & $118_{-40}^{+38}$ \\
\hline
\end{tabular}

disc is truncated, the strength of the reprocessed features (iron line and Compton reflection continuum) are reduced because of the smaller solid angle subtended by the matter to the primary X-ray source; the line width is also expected to decrease, because the Doppler and Gravitational broadenings are most relevant at small radii. The amount of thermal disc emission is also reduced, and the hard X-ray spectrum is flattened due to the less effective cooling. It is worth noting that these relations between $\Gamma$ (the power law spectral index) and the reprocessed features are not expected in the ionization scenario, where the reduction of the line $E W$ due to resonant trappings or full matter ionization is not accompanied by a reduction of the thermal disc emission and therefore of the cooling in the Comptonizing corona.

In this paper we present possible evidence for a changing inner radius in the broad line radio-quiet quasar Q0056-363 $(z=0.162)$. The source was observed by XMM-Newton twice. In the first observation, performed on July 2000 and about $20 \mathrm{ks}$ long, an intense ( $E W$ of about $250 \mathrm{eV}$ ) and broad (velocity width of about $25000 \mathrm{~km} \mathrm{~s}^{-1}$ ) iron line was present, strongly suggesting emission from the innermost regions of the accretion disc (Porquet \& Reeves 2003). Q0056-363 is presently the most luminous quasar with an observed relativistic line. The source was then reobserved on December 21-23, 2003 for about $90 \mathrm{ks}$. Here we report on the latter observation, and the comparison between the two.

\section{Data reduction and analysis}

Both XMM-Newton observations of Q0056-363 were reduced with SAS 6.0.0, following standard procedures and screening periods of high background flaring according to the method presented in Piconcelli et al. (2004), based on the cumulative distribution function of background lightcurve count rates. This method, which maximes the signal-to-noise ratio, resulted in net exposure times of $\simeq 14 \mathrm{ks}$ and $\simeq 94 \mathrm{ks}$, for the 2001 and the 2003 observation, respectively. EPIC PN (Strüder et al. 2001) and MOS (Turner et al. 2001) spectra were extracted, adopting circular extraction regions with radii of 40 and 45 arcsec, respectively. After inspecting the results from the SAS tool EPATPLOT and from some fits on single and double pattern spectra separately, we concluded that the effect of pileup is negligible in both observations, allowing us to use pattern 0-4 and $0-12$ for the PN and the MOS. Due to operational needs, the first MOS observation was splitted in two $\simeq 5 \mathrm{ks}$ segments, with different filters. In both observations, the addition of the MOS in the spectral fits do not significantly increase the precision with which spectral parameters are determined. For these reasons, and for the sake of simplicity, we will use and discuss in the following only results from the PN, unless explicitely stated.

For spectral fitting, the spectra were rebinned in order to have at least 25 counts per bin and to oversample the energy resolution by at least a factor of 3 . Spectral fits are performed with the software package XSPEC. All reported errors will correspond to $90 \%$ confidence level for one interesting parameter $\left(\Delta \chi^{2}=2.71\right)$.

\section{Results}

\subsection{Comparison between the 2000 and 2003 observations}

In both observations, a simple absorbed power law (with Galactic absorption fixed at $\left.1.94 \times 10^{20} \mathrm{~cm}^{-2}\right)^{1}$ fails to fit the data, while the inclusion of a soft excess and an iron line are sufficient to reasonably model the spectra. For the sake of simplicity, and because we are interested here mainly to the comparison between the 2000 and 2003 spectra, rather than to any detailed modeling, we parametrized the soft excess with a simple power law, and the iron line with a Gaussian with centroid energy fixed at $6.4 \mathrm{keV}$ (rest frame) and $\sigma$ as a free parameter. This model provides good enough fits over the $0.5-12 \mathrm{keV}$ energy band ( $\chi^{2} /$ d.o.f. of $127.3 / 127$ and $359.4 / 223$ for the 2000 and 2003 observations, respectively). The best fit parameters are reported in Table 1.

While the full band fluxes in the two observations are rather similar, spectral changes are apparent. In the 2000 observation, both the soft and hard power laws are significantly steeper than in the 2003 observation, while the iron line is more intense (even if at the $90 \%$ confidence level the EWs are only just inconsistent each other). The width of the line is instead poorly constrained. In Fig. 1, the $2-10 \mathrm{keV}$ spectra and residuals, when fitting with a simple power law, are shown. An eye inspection would tell that the line in the 2000 is broader, but the quality of the data, especially for the 2000 observation, is not good enough to make this difference statistically significant.

Similar results are obtained leaving the line centroid energy free to vary. The centroid energy, $\sigma$ and $E W$ are: $6.31_{-0.10}^{+0.19} \mathrm{keV}$, $270_{-100}^{+130} \mathrm{eV}, 240_{-80}^{+112} \mathrm{eV}(2000)$, and $6.47_{-0.09}^{+0.09} \mathrm{keV}, 220_{-80}^{+100} \mathrm{eV}$, $109_{-32}^{+39} \mathrm{eV}$ (2003). The statistical quality of the fits did not improve.

If the line is fitted with a relativistic profile in Schwarzschild metric, with fixed centroid energy $(6.4 \mathrm{keV})$

\footnotetext{
1 http://heasarc.gsfc.nasa.gov/cgi-bin/Tools/w3nh/ w3nh.pl
} 
2000

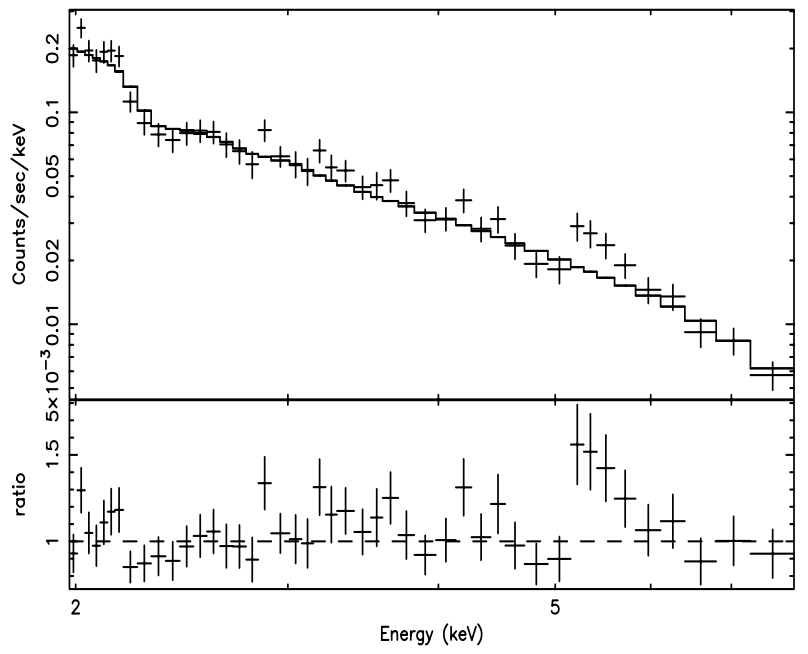

2003

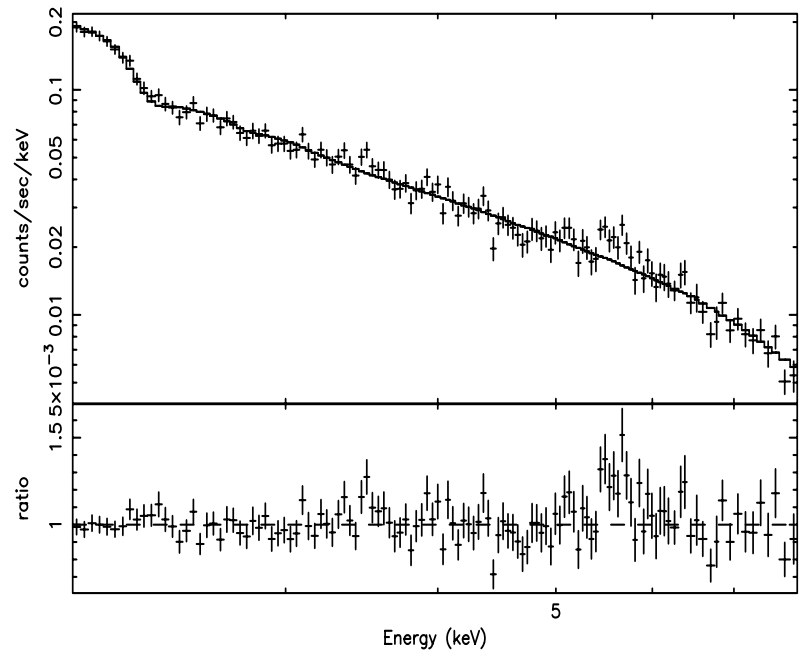

Fig. 1. Left panel: data, best fit model and data/model ratio in the iron line region for the 2000 observation, when fitted with a simple power law. Right panel: the same, but for the 2003 observation.

outer radius $\left(1000 r_{\mathrm{g}}\right)$, inclination angle $\left(30^{\circ}\right)$, and emissivity law (power law with index -2 ), the best fit inner radius is $37_{-29}^{+47} r_{\mathrm{g}}$ for the 2003 observation, and basically unconstrained $\left(r<100 r_{\mathrm{g}}\right)$ for the 2000 observation. In both cases, the quality of the fit is similar to those with a Gaussian line. Again, the results did not change significantly leaving the centroid energy free to vary.

To illustrate the spectral changes, in Fig. 2 we show the 2003 spectrum and residuals when fitted with the 2000 best fit model (no renormalization is made). An excess of photons at high energies, and an even more dramatic deficit at low energies are indeed apparent, along with a deficit at the iron line energies. In the soft band $(0.5-2 \mathrm{keV})$ the flux in 2000 is higher than in 2003 by about $13 \%$, while in the hard band $(2-10 \mathrm{keV})$ the difference is very small, only $3 \%$. The UV flux, measured with the OM, also changed between the two observations, being about 1.6 times larger in 2000 (see Table 1). All these numbers indicate flux variations increasing in amplitude with decreasing photon frequency.

\subsection{Time-resolved analysis of the 2003 observation}

We also searched for short term spectral changes, by dividing the 2003 observation in 5 intervals. No statistically significant changes, either in the continuum or line properties, were found.

We also searched for transient emission and absorption features, as those recently observed in many sources (see e.g. Porquet et al. 2004; Dovčiak et al. 2004, and references therein). We set, rather arbitrarily, a confidence level of $99 \%$ (according to the F-test) as the detection threshold for a feature. An absorption feature in the third interval has been found to meet this criterion (see Fig. 3). If fitted with a Gaussian with $\sigma$ fixed to 0.1 , the centroid energy is $5.34_{-0.13}^{+0.12} \mathrm{keV}$ (rest frame) and the $E W$ is $-75_{-41}^{+34} \mathrm{eV}$. The significance of this feature is $99.2 \%$. Leaving $\sigma$ free to vary, we do not obtain a significantly better fit. In the MOS, this feature is neither required nor ruled out. As a word of caution, we note that a possible emission line

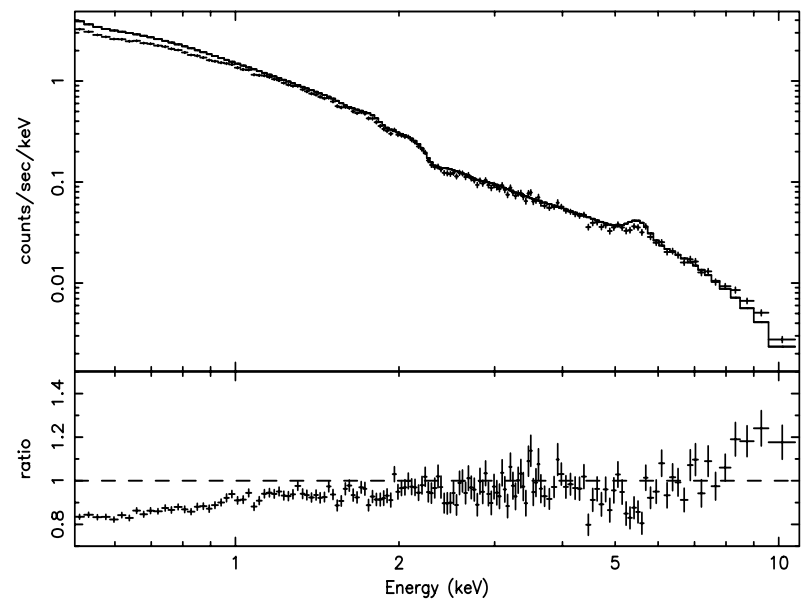

Fig. 2. The 2003 spectrum when fitted with the 2000 best fit model. Note the harder spectrum, both at low and high energies, and a deficit at the iron line energies ( $5.5 \mathrm{keV}$ in the observed frame), due to the fact that in the 2000 observation the line is more intense.

at about $3.7 \mathrm{keV}$ (observed frame) could also be present, but having a significance of $98.7 \%$ is just below our threshold.

\section{Discussion}

The spectral changes described in the previous section are consistent with a "truncating disc" scenario in which the disc inner radius was closer to the $\mathrm{BH}$ in 2000 while the disc was truncated in 2003. The smaller soft excess, lower UV flux, flatter hard X-ray spectrum, fainter iron line in the latter observation can all be explained in this way, at least qualitatively. In fact, in a truncated disc the thermal emission (likely responsible for the UV and soft X-ray emission) is obviously smaller, the iron line is less intense (due to the smaller solid angle subtended by the disc to the primary X-ray emitting region) and less broad (because the relativistic effects are less important), and the hard X-ray spectrum is flatter due to the less effective cooling. 


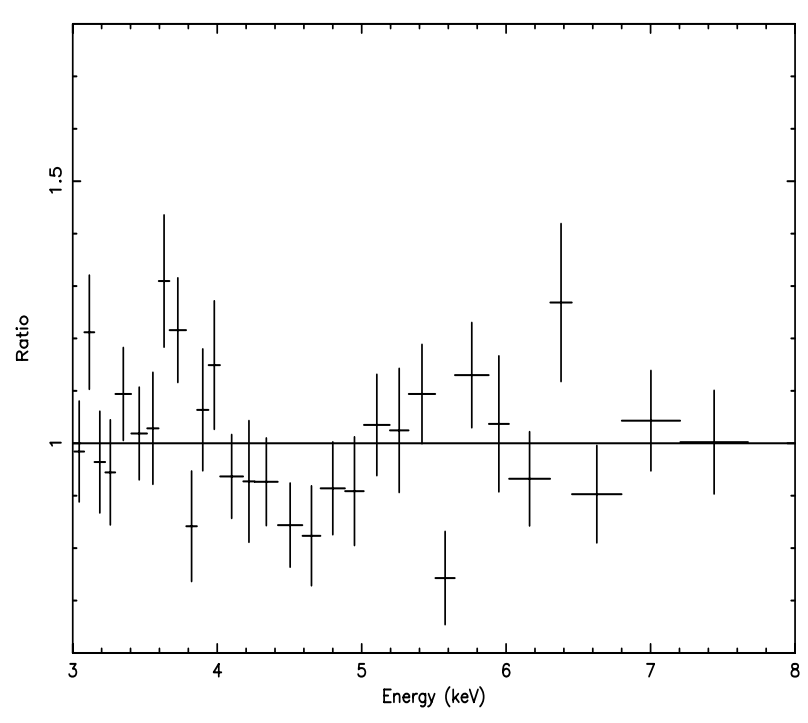

Fig. 3. The spectrum of the third interval in the 2003 observation. An absorption feature at $\sim 4.5 \mathrm{keV}$ (observer frame) is apparent.

A strong prediction of this scenario is a narrower iron line in the 2003 observation. Unfortunately, the quality of the spectra is not good enough to draw any conclusion in this respect, and for the time being disc truncation must be considered only a plausible and appealing hypothesis. Giving its potential interest, however, we discuss this possibility more extensively.

Disc truncation has been proposed by Zdziarski et al. (1999) to explain the observed correlation between the strength of the Compton Reflection component and the spectral slope in AGN and Galactic Black Hole systems, and by Lubinski \& Zdziarski (2001) to explain the correlation between the iron line width and $E W$ and the slope.

In Galactic Black Hole systems, disc emptying and refilling is believed to play an important role, and this phenomenon seems to be strongly related to matter ejection (e.g. Fender et al. 2004, and references therein). In fact ejection of matter is usually associated with Hard X-ray states both at relatively low and very high accretion rates (e.g. Fender et al. 2004). In the Low/Hard state, where $\dot{m}$ is $\lesssim 1 \%$ the Eddington rate, the ejection is in form of a steady jet (Gallo et al. 2003), and the innermost regions of the accretion disc is absent. In the High/Soft state (high $\dot{m}$ ), the disc extends down to the innermost stable orbit; radio emission is suppressed, suggesting the quenching of matter ejection. During the transition from Low to High states, there is a phase (called Very High State) in which the disc is refilling, the hard (i.e. RXTE-PCA band) X-ray luminosity is quite stable at large values but with still a hard spectrum (continuously softening until the High state is reached; see Fig. 7 of Fender et al. 2004), and there are episodes of high velocity ejections. It is also worth noting that some sources (most notably GRS 1915+105, Fender \& Belloni 2004 and references therein) switches between High and Very High states only (with corresponding variations of the inner disc radius) without going through the entire cycle. If this scenario is, at least qualitatively, applicable also to AGN (as suggested by the scaling of the radio/X-ray coupling across a range of $\gtrsim 10^{7}$ solar masses, Merloni et al. 2003; Maccarone et al. 2003), and given the fact that $\dot{m}$ seems to be large in both observations (Porquet \& Reeves 2003 estimated $L / L_{\text {edd }} \sim 0.6-0.8$, and in the 2003 observation the hard X-ray flux is similar), one can speculate that the source was in a High State in 2000, and in a Very High State in 2003.

In this scenario, we can also fit the absorption feature at about $5.3 \mathrm{keV}$ (rest frame) possibly detected in the spectrum of the third interval in which we divided the 2003 observation. The most natural interpretation is in term of a redshifted iron line. If entirely due to Doppler effect, this implies $v=0.2 c$ if the line is the He-like, or $v=0.23 c$ if the line is the H-like one. If instead the redshift is entirely gravitational, this implies (in Schwarzschild metric) a location of the absorbing matter at $r \sim 5.5$ or $4.9 r_{\mathrm{g}}$ (assuming that the primary X-rays are produced very close to be blob, so that there is no shift between the two regions). The equivalent width is pretty large, implying a large column density (at least $10^{23} \mathrm{~cm}^{-2}$ ) and either a significant iron overabundance or a strong contribution from turbulence (Bianchi et al. 2005).

Iron redshifted absorption features have possibly been already observed in a few objects (e.g. Nandra et al. 1999; Longinotti et al. 2003). Interestingly, Dadina et al. (2005) have found evidence for transient redshifted iron lines in the BeppoSAX spectrum of Mrk 509, both red- and blue-shifted. It is worth noting that transient and shifted absorption lines are naturally expected in models involving blobby ejection and downfalling of matter, as in the recently proposed aborted-jet model of Ghisellini et al. (2004), where at least part of the heating of the X-ray emitting corona is due to collision by blobs ejected with a velocity smaller than the escape velocity. Absorption by one of these blobs, either downfalling (the $\sim 20 \mathrm{ks}$ observing time corresponds, for a velocity of about $0.2 c$ and a Black Hole of about $5 \times 10^{8} M_{\odot}$, to a crossing distance of about a Schwarzschild radius) or very close to the $\mathrm{BH}$, and then gravitationally redshifted, could explain our findings. If, as in Galactic Black Hole systems, ejection of matter preferentially occurs when the accretion disc is truncated, it is not surprising to find such a feature in the 2003 observation.

\section{Conclusions}

Comparing the 2000 and 2003 observations of the quasar, Q0056-363, we have found differences which can be interpreted as a change of the disc inner radius, which was possibly smaller (i.e. closer to the Black Hole event horizon) in the former observation. In the latter observation we have also found evidence, even if marginal, for a transient absorption line, interpreted as redshifted ionized iron. Intriguingly, these findings can be qualitatively fitted into the scenario proposed for stellar mass Black Hole systems (Fender et al. 2004), thus possibly providing a link between properties of accreting Black Holes accross a range of masses of several orders of magnitude.

Acknowledgements. We thank Elena Gallo for enlightening discussions, and the anonymous referee for useful comments. G.M. acknowledges financial support from MIUR under grant PRIN-03-0223. This paper is based on observations obtained with XMM-Newton, 
an ESA science mission with instruments and contributions directly funded by ESA Member States and the USA (NASA).

\section{References}

Belloni, T., Mendez, M., King, A. R., et al. 1997, ApJ, 488, L109 Bianchi, S., Matt, G., Balestra, I., Guainazzi, M., \& Perola, G. C. 2004, A\&A, 422, 65

Bianchi, S., Matt, G., Nicastro, F., Porquet, D., \& Dubau, J. 2005, MNRAS, in press

Dadina, M., Cappi, M., Malaguti, G., Ponti, G., \& De Rosa, A. 2005, A\&A, submitted

Done, C., \& Gierlinski, M. 2004, Prog. Theor. Phys., 155, 9

Dovčiak, M., Bianchi, S., Guainazzi, M., Karas, V., \& Matt, G. 2004, MNRAS, 350, 745

Fabian, A. C., Vaughan, S., Nandra, K., et al. 2002, MNRAS, 335, L1

Fender, R. P., \& Belloni, T. 2004, ARA\&A, 42, 317

Fender, R. P., Belloni, T., \& Gallo, E. 2004, MNRAS, 355, 1105

Gallo, E., Fender, R. P., \& Pooley, G. G. 2003, MNRAS, 344, 60

Ghisellini, G., Haardt, F., \& Matt, G. 2004, A\&A, 413, 535
Longinotti, A. L., Cappi, M., Nandra, K., Dadina, M., \& Pellegrini, S. 2003, A\&A, 410, 471

Lubinski, P., \& Zdziarski, A. A. 2001, MNRAS, 323, L37

Maccarone, T. J., Gallo, E., \& Fender, R. 2003, MNRAS, 345, L19

Marscher, A. P., Jorstad, S. G., Gomez, J.-L., et al. 2002, Nature, 417, 625

Matt, G., Fabian, A. C., \& Ross, R. R. 1996, MNRAS, 278, 1111

Merloni, A., Heinz, S., \& Di Matteo, T. 2003, MNRAS, 345, 1057

Nandra, K., George, I. M., Mushotzky, R. F., Turner, T. J., \& Yaqoob, T. 1999, ApJ, 523, L17

Piconcelli, E., Jimenez-Bailón, E., Guainazzi, M., et al. 2004, MNRAS, 351, 161

Porquet, D., \& Reeves, J. N. 2003, A\&A, 408, 119

Porquet, D., Reeves, J. N., Uttley, P., \& Turner, T. J. 2004, A\&A, 427, 101

Strüder, L., Briel, U., Dennerl, K., et al. 2001, A\&A, 365, L18

Turner, M. J. L., Abbey, A., Arnaud, M., et al. 2001, A\&A, 365, L27

Turner, T. J., Mushotzky, R. F., Yaqoob, T., et al. 2002, ApJ, 574, L123

Zdziarski, A. A., Lubinski, P., \& Smith, D. A. 1999, MNRAS, 303, L11 